\title{
The Product Space of Real Normed Spaces and its Properties
}

\author{
Noboru Endou \\ Gifu National College of Technology \\ Japan \\ Keiichi Miyajima \\ Ibaraki University \\ Hitachi, Japan
}

Yasunari Shidama

Shinshu University

Nagano, Japan

\begin{abstract}
Summary. In this article, we define the product space of real linear spaces and real normed spaces. We also describe properties of these spaces.
\end{abstract}

MML identifier: PRVECT_2, version: 7.8.05 4.84.971

The terminology and notation used here are introduced in the following articles: [20], [9], [22], [2], [1], [19], [5], [23], [7], [10], [8], [4], [13], [12], [21], [14], [3], [6], [16], [11], [15], [17], and [18].

\section{The Product Space of Real Linear Spaces}

The following propositions are true:

(1) Let $s, t$ be sequences of real numbers and $g$ be a real number. Suppose that for every element $n$ of $\mathbb{N}$ holds $t(n)=|s(n)-g|$. Then $s$ is convergent and $\lim s=g$ if and only if $t$ is convergent and $\lim t=0$.

(2) Let $x, y$ be finite sequences of elements of $\mathbb{R}$. Suppose len $x=$ len $y$ and for every element $i$ of $\mathbb{N}$ such that $i \in \operatorname{Seg}$ len $x$ holds $0 \leq x(i)$ and $x(i) \leq y(i)$. Then $|x| \leq|y|$.

(3) Let $F$ be a finite sequence of elements of $\mathbb{R}$. If for every element $i$ of $\mathbb{N}$ such that $i \in \operatorname{dom} F$ holds $F(i)=0$, then $\sum F=0$. 
Let $f$ be a function and let $X$ be a set. A function is called a multi-operation of $X$ and $f$ if:

(Def. 1) $\operatorname{dom}$ it $=\operatorname{dom} f$ and for every set $i$ such that $i \in \operatorname{dom} f$ holds $\operatorname{it}(i)$ is a function from [: $X, f(i)$ : into $f(i)$.

Let $F$ be a sequence of non empty sets and let $X$ be a set. Observe that every multi-operation of $X$ and $F$ is finite sequence-like.

We now state the proposition

(4) Let $X$ be a set, $F$ be a sequence of non empty sets, and $p$ be a finite sequence. Then $p$ is a multi-operation of $X$ and $F$ if and only if len $p=$ len $F$ and for every set $i$ such that $i \in \operatorname{dom} F$ holds $p(i)$ is a function from [: $X, F(i)$ : into $F(i)$.

Let $F$ be a sequence of non empty sets, let $X$ be a set, let $p$ be a multioperation of $X$ and $F$, and let $i$ be an element of $\operatorname{dom} F$. Then $p(i)$ is a function from [: $X, F(i)$ : into $F(i)$.

Next we state the proposition

(5) Let $X$ be a non empty set, $F$ be a sequence of non empty sets, and $f$, $g$ be functions from $\left[: X, \prod F\right.$ : into $\prod F$. Suppose that for every element $x$ of $X$ and for every element $d$ of $\prod F$ and for every element $i$ of $\operatorname{dom} F$ holds $f(x, d)(i)=g(x, d)(i)$. Then $f=g$.

Let $F$ be a sequence of non empty sets, let $X$ be a non empty set, and let $p$ be a multi-operation of $X$ and $F$. The functor $\prod^{\circ} p$ yielding a function from $\left[: X, \prod F:\right.$ into $\prod F$ is defined as follows:

(Def. 2) For every element $x$ of $X$ and for every element $d$ of $\prod F$ and for every element $i$ of $\operatorname{dom} F$ holds $\left(\prod^{\circ} p\right)(x, d)(i)=p(i)(x, d(i))$.

Let $R$ be a binary relation. We say that $R$ is real-linear-space-yielding if and only if:

(Def. 3) For every set $S$ such that $S \in \operatorname{rng} R$ holds $S$ is a real linear space.

Let us note that there exists a finite sequence which is non empty and reallinear-space-yielding.

A real linear space-sequence is a non empty real-linear-space-yielding finite sequence.

Let $G$ be a real linear space-sequence and let $j$ be an element of $\operatorname{dom} G$. Then $G(j)$ is a real linear space.

Let $G$ be a real linear space-sequence. The functor $\bar{G}$ yielding a sequence of non empty sets is defined by:

(Def. 4) len $\bar{G}=\operatorname{len} G$ and for every element $j$ of $\operatorname{dom} G$ holds $\bar{G}(j)=$ the carrier of $G(j)$.

Let $G$ be a real linear space-sequence and let $j$ be an element of $\operatorname{dom} \bar{G}$. Then $G(j)$ is a real linear space. 
Let $G$ be a real linear space-sequence. The functor $\left\langle{ }_{G_{i}}\right\rangle_{i}$ yielding a family of binary operations of $\bar{G}$ is defined as follows:

(Def. 5) $\operatorname{len}\left(\left\langle+_{G_{i}}\right\rangle_{i}\right)=\operatorname{len} \bar{G}$ and for every element $j$ of $\operatorname{dom} \bar{G}$ holds $\left\langle+_{G_{i}}\right\rangle_{i}(j)=$ the addition of $G(j)$.

The functor $\left\langle-G_{i}\right\rangle_{i}$ yields a family of unary operations of $\bar{G}$ and is defined as follows:

(Def. 6) $\operatorname{len}\left(\left\langle-G_{i}\right\rangle_{i}\right)=\operatorname{len} \bar{G}$ and for every element $j$ of $\operatorname{dom} \bar{G}$ holds $\left\langle-G_{i}\right\rangle_{i}(j)=$ comp $G(j)$.

The functor $\left\langle 0_{G_{i}}\right\rangle_{i}$ yielding an element of $\prod \bar{G}$ is defined by:

(Def. 7) For every element $j$ of $\operatorname{dom} \bar{G}$ holds $\left\langle 0_{G_{i}}\right\rangle_{i}(j)=$ the zero of $G(j)$.

The functor multop $G$ yields a multi-operation of $\mathbb{R}$ and $\bar{G}$ and is defined by:

(Def. 8) len multop $G=\operatorname{len} \bar{G}$ and for every element $j$ of $\operatorname{dom} \bar{G}$ holds $($ multop $G)(j)=$ the external multiplication of $G(j)$.

Let $G$ be a real linear space-sequence. The functor $\prod G$ yielding a strict non empty RLS structure is defined by:

(Def. 9) $\Pi G=\left\langle\prod \bar{G},\left\langle 0_{G_{i}}\right\rangle_{i}, \prod^{\circ}\left(\left\langle+_{G_{i}}\right\rangle_{i}\right), \prod^{\circ} \operatorname{multop} G\right\rangle$.

Let $G$ be a real linear space-sequence. One can check that $\prod G$ is Abelian, add-associative, right zeroed, right complementable, and real linear space-like.

\section{The Product Space of Real Normed Spaces}

Let $R$ be a binary relation. We say that $R$ is real-norm-space-yielding if and only if:

(Def. 10) For every set $x$ such that $x \in \operatorname{rng} R$ holds $x$ is a real normed space.

One can check that there exists a finite sequence which is non empty and real-norm-space-yielding.

A real norm space-sequence is a non empty real-norm-space-yielding finite sequence.

Let $G$ be a real norm space-sequence and let $j$ be an element of $\operatorname{dom} G$. Then $G(j)$ is a real normed space.

Let us note that every finite sequence which is real-norm-space-yielding is also real-linear-space-yielding.

Let $G$ be a real norm space-sequence and let $x$ be an element of $\prod \bar{G}$. The functor normsequence $(G, x)$ yields an element of $\mathcal{R}^{\operatorname{len} G}$ and is defined as follows:

(Def. 11) len normsequence $(G, x)=\operatorname{len} G$ and for every element $j$ of $\operatorname{dom} G$ holds (normsequence $(G, x))(j)=($ the norm of $G(j))(x(j))$.

Let $G$ be a real norm space-sequence. The functor productnorm $G$ yields a function from $\prod \overline{(G \text { qua real linear space-sequence })}$ into $\mathbb{R}$ and is defined by:

(Def. 12) For every element $x$ of $\prod \bar{G}$ holds

$($ productnorm $G)(x)=\mid$ normsequence $(G, x) \mid$. 
Let $G$ be a real norm space-sequence. The functor $\prod G$ yielding a strict non empty normed structure is defined as follows:

(Def. 13) The RLS structure of $\prod G=\prod(G$ qua real linear space-sequence) and the norm of $\prod G=$ productnorm $G$.

In the sequel $G$ is a real norm space-sequence.

We now state four propositions:

(6) $\prod G=\left\langle\prod \bar{G},\left\langle 0_{G_{i}}\right\rangle_{i}, \Pi^{\circ}\left(\left\langle+_{G_{i}}\right\rangle_{i}\right), \Pi^{\circ}\right.$ multop $G$, productnorm $\left.G\right\rangle$.

(7) For every vector $x$ of $\prod G$ and for every element $y$ of $\prod \bar{G}$ such that $x=y$ holds $\|x\|=\mid$ normsequence $(G, y) \mid$.

(8) For all elements $x, y, z$ of $\prod \bar{G}$ and for every element $i$ of $\mathbb{N}$ such that $i \in \operatorname{dom} x$ and $z=\left(\Pi^{\circ}\left(\left\langle+_{G_{i}}\right\rangle_{i}\right)\right)(x, y)$ holds (normsequence $\left.(G, z)\right)(i) \leq$ (normsequence $(G, x)+$ normsequence $(G, y))(i)$.

(9) For every element $x$ of $\prod \bar{G}$ and for every element $i$ of $\mathbb{N}$ such that $i \in \operatorname{dom} x$ holds $0 \leq$ (normsequence $(G, x))(i)$.

Let $G$ be a real norm space-sequence. Observe that $\prod G$ is real normed space-like, real linear space-like, Abelian, add-associative, right zeroed, and right complementable.

One can prove the following propositions:

(10) Let $G$ be a real norm space-sequence, $i$ be an element of $\operatorname{dom} G, x$ be a point of $\prod G, y$ be an element of $\prod \bar{G}$, and $x_{1}$ be a point of $G(i)$. If $y=x$ and $x_{1}=y(i)$, then $\left\|x_{1}\right\| \leq\|x\|$.

(11) Let $G$ be a real norm space-sequence, $i$ be an element of $\operatorname{dom} G, x, y$ be points of $\prod G, x_{1}, y_{1}$ be points of $G(i)$, and $z_{1}, z_{2}$ be elements of $\prod \bar{G}$. If $x_{1}=z_{1}(i)$ and $z_{1}=x$ and $y_{1}=z_{2}(i)$ and $z_{2}=y$, then $\left\|y_{1}-x_{1}\right\| \leq\|y-x\|$.

(12) Let $G$ be a real norm space-sequence, $s_{1}$ be a sequence of $\prod G, x_{0}$ be a point of $\prod G$, and $y_{0}$ be an element of $\prod \bar{G}$. Suppose $x_{0}=y_{0}$ and $s_{1}$ is convergent and $\lim s_{1}=x_{0}$. Let $i$ be an element of $\operatorname{dom} G$. Then there exists a sequence $s_{2}$ of $G(i)$ such that $s_{2}$ is convergent and $y_{0}(i)=\lim s_{2}$ and for every element $m$ of $\mathbb{N}$ there exists an element $s_{3}$ of $\prod \bar{G}$ such that $s_{3}=s_{1}(m)$ and $s_{2}(m)=s_{3}(i)$.

(13) Let $G$ be a real norm space-sequence, $s_{1}$ be a sequence of $\prod G, x_{0}$ be a point of $\prod G$, and $y_{0}$ be an element of $\prod \bar{G}$. Suppose that

(i) $x_{0}=y_{0}$, and

(ii) for every element $i$ of $\operatorname{dom} G$ there exists a sequence $s_{2}$ of $G(i)$ such that $s_{2}$ is convergent and $y_{0}(i)=\lim s_{2}$ and for every element $m$ of $\mathbb{N}$ there exists an element $s_{3}$ of $\prod \bar{G}$ such that $s_{3}=s_{1}(m)$ and $s_{2}(m)=s_{3}(i)$.

Then $s_{1}$ is convergent and $\lim s_{1}=x_{0}$.

(14) For every real norm space-sequence $G$ such that for every element $i$ of dom $G$ holds $G(i)$ is complete holds $\prod G$ is complete. 


\section{REFERENCES}

[1] Grzegorz Bancerek. König's theorem. Formalized Mathematics, 1(3):589-593, 1990.

[2] Grzegorz Bancerek. The ordinal numbers. Formalized Mathematics, 1(1):91-96, 1990.

[3] Grzegorz Bancerek and Krzysztof Hryniewiecki. Segments of natural numbers and finite sequences. Formalized Mathematics, 1(1):107-114, 1990.

[4] Czesław Byliński. Binary operations. Formalized Mathematics, 1(1):175-180, 1990.

[5] Czesław Byliński. The complex numbers. Formalized Mathematics, 1(3):507-513, 1990.

[6] Czesław Byliński. Finite sequences and tuples of elements of a non-empty sets. Formalized Mathematics, 1(3):529-536, 1990.

[7] Czesław Byliński. Functions and their basic properties. Formalized Mathematics, 1(1):5565, 1990.

[8] Czesław Byliński. Functions from a set to a set. Formalized Mathematics, 1(1):153-164, 1990.

[9] Czesław Byliński. Some basic properties of sets. Formalized Mathematics, 1(1):47-53, 1990.

[10] Czesław Byliński. The sum and product of finite sequences of real numbers. Formalized Mathematics, 1(4):661-668, 1990.

[11] Agata Darmochwał. The Euclidean space. Formalized Mathematics, 2(4):599-603, 1991.

[12] Jarosław Kotowicz. Convergent sequences and the limit of sequences. Formalized Mathematics, 1(2):273-275, 1990.

[13] Jarosław Kotowicz. Real sequences and basic operations on them. Formalized Mathematics, 1(2):269-272, 1990.

[14] Eugeniusz Kusak, Wojciech Leończuk, and Michał Muzalewski. Abelian groups, fields and vector spaces. Formalized Mathematics, 1(2):335-342, 1990.

[15] Anna Lango and Grzegorz Bancerek. Product of families of groups and vector spaces. Formalized Mathematics, 3(2):235-240, 1992.

[16] Beata Padlewska and Agata Darmochwał. Topological spaces and continuous functions. Formalized Mathematics, 1(1):223-230, 1990.

[17] Jan Popiołek. Real normed space. Formalized Mathematics, 2(1):111-115, 1991.

[18] Yasunari Shidama. Banach space of bounded linear operators. Formalized Mathematics, 12(1):39-48, 2004.

[19] Andrzej Trybulec. Subsets of complex numbers. To appear in Formalized Mathematics.

[20] Andrzej Trybulec. Tarski Grothendieck set theory. Formalized Mathematics, 1(1):9-11, 1990.

[21] Wojciech A. Trybulec. Vectors in real linear space. Formalized Mathematics, 1(2):291296, 1990.

[22] Zinaida Trybulec. Properties of subsets. Formalized Mathematics, 1(1):67-71, 1990.

[23] Edmund Woronowicz. Relations and their basic properties. Formalized Mathematics, 1(1):73-83, 1990. 